\title{
Dispersion error of a beam splitter cube in white-light spectral interferometry
}

\author{
P. HLUBINA*, J. LUŇÁČEK, D. CIPRIAN, and R. CHLEBUS \\ Department of Physics, Technical University Ostrava, 17 listopadu 15, 70833 Ostrava-Poruba, \\ Czech Republic
}

\begin{abstract}
We revealed that the phase function of a thin-film structure measured by a white-light spectral interferometric technique depends on the path length difference adjusted in a Michelson interferometer. This phenomenon is due to a dispersion error of a beam splitter cube, the effective thickness of which varies with the adjusted path length difference. A technique for eliminating the effect in measurement of the phase function is described. In a first step, the Michelson interferometer with same metallic mirrors is used to measure the effective thickness of the beam splitter cube as a function of the path length difference. In a second step, one of the mirrors of the interferometer is replaced by a thin-film structure and its phase function is measured for the same path length differences as those adjusted in the first step. In both steps, the phase is retrieved from the recorded spectral interferograms by using a windowed Fourier transform applied in the wavelength domain.
\end{abstract}

Keywords: spectral interferometry, white-light source, Michelson interferometer, beam splitter cube, dispersion, BK7 glass, phase retrieval, effective thickness.

\section{Introduction}

White-light spectral interferometry based on channelled spectrum detection and utilizing a broadband source in combination with a standard Michelson interferometer has been widely used for distance and displacement measurements [1-3], in optical profilometry [4-7] and for material characterization [8-13].

Michelson interferometers that split light into two beams and recombine them to produce the interference pattern comprise beam splitters that are typically constructed of dispersive materials such as optical glasses. Beam splitter cubes are widely used to ensure matching of geometrical path lengths of light rays in the two arms of the interferometer. However, commercially available beam splitters have a dispersion error [14-16] due to different geometrical path lengths of the light rays in dispersive glass in the two interferometer arms. To reduce the dispersion error as much as possible, two degrees of freedom must be controlled during the fabrication process [16]. They include the lateral position and relative rotation of the two prisms that comprise the beam splitter cube. Recently, the effective thickness of the beam splitter was introduced [17] to quantify the dispersion error as a result of the asymmetry in the interferometer. Most recently, knowledge of the effective thickness was used in employment of a new method for measuring a phase function of a thin-film structure [11].

\footnotetext{
*e-mail: petr.hlubina@vsb.cz
}

The aim of the paper is to describe a white-light spectral interferometric technique that can be used to eliminate the dependence of the phase function of a thin-film structure on the path length difference adjusted in a Michelson interferometer. This dependence is due to a dispersion error of a beam splitter cube, the effective thickness of which varies with the adjusted path length difference. First, the Michelson interferometer with same metallic mirrors is used to measure the effective thickness of the beam splitter as a function of the path length difference. The effective thickness is given by the slope of the dependence of the phase on the refractive index of BK7 glass [13] from which the beam splitter cube is made. Second, one of the mirrors of the Michelson interferometer is replaced by a thin-film structure and its phase function is measured for the same path length differences as those adjusted in the first step. In both steps, the phase is retrieved from the recorded spectral interferograms by using a windowed Fourier transform (WFT) applied in the wavelength domain [13].

\section{Theory}

\subsection{Michelson interferometer with two identical metallic mirrors}

Let us consider the mutual interference of two beams from a broadband source at the output of a Michelson interferometer with two identical metallic mirrors and a beam splitter cube of the effective thickness $t_{\text {eff }}$ [17]. We assume that the geometrical path lengths of the light rays in dispersive glass of the beam splitter are not the same for both 


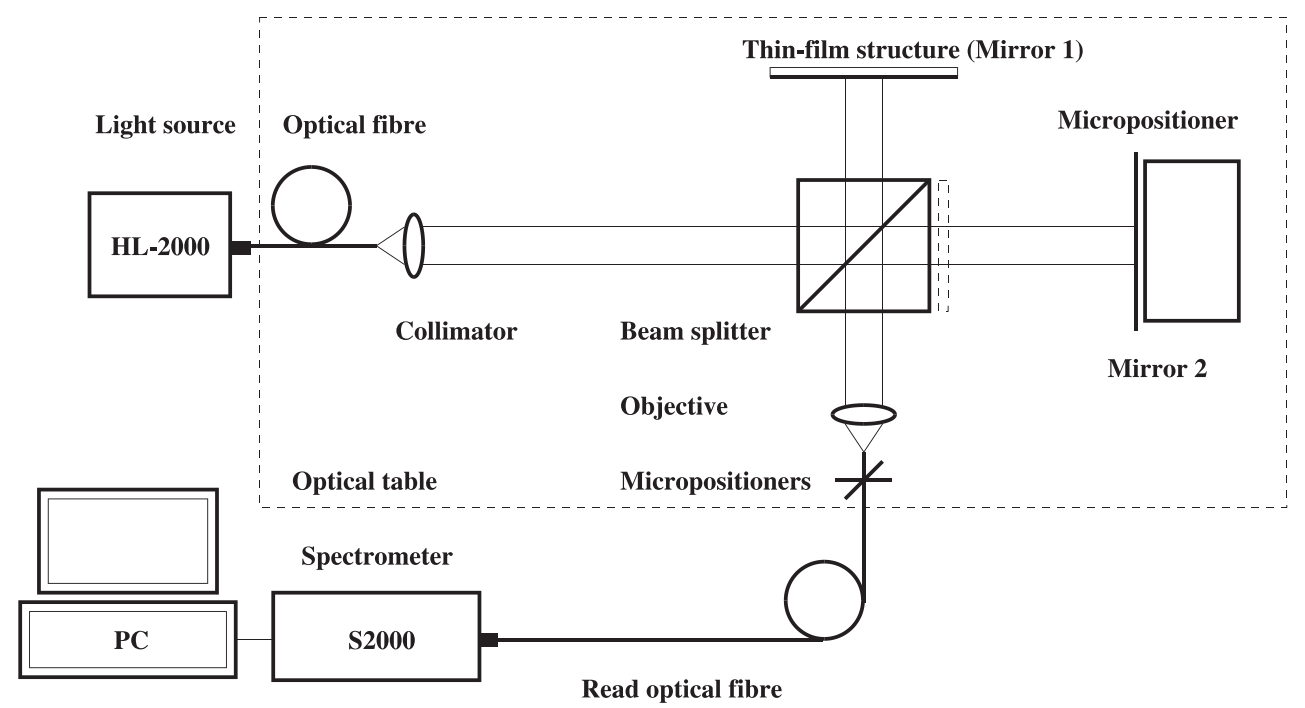

Fig. 1. Experimental set-up of a Michelson interferometer with a beam splitter cube of given effective thickness.

interferometer arms so that the beam splitter can be represented by an ideal beam splitter and a plate of the same dispersion and of the thickness $t_{\text {eff }}$ (see Fig. 1).

We are interesting in the spectral interference signal $S_{M}(\lambda)$ defined as [11]

$$
S_{M}(\lambda)=I_{M}(\lambda) / I_{M}^{0}(\lambda)-1,
$$

where $I_{M}(\lambda)$ is the spectral intensity (channelled spectrum) and $I_{M}^{0}(\lambda)$ is the reference (unmodulated) spectrum. If both spectra are recorded at the output of the interferometer by a fibre-optic spectrometer of a Gaussian response function, the spectral interference signal can be expressed as [3]

$$
\begin{aligned}
S_{M}(\lambda)= & V_{I} \exp \left\{-\left(\pi^{2} / 2\right)\left[\Delta_{M}^{g}(\lambda) \Delta \lambda_{R} / \lambda^{2}\right]\right\}, \\
& \times \cos \left[(2 \pi / \lambda) \Delta_{M}(\lambda)\right]
\end{aligned}
$$

where $V_{I}$ is the visibility term, $\Delta \lambda_{R}$ denotes the width of the spectrometer response function, and $\Delta_{M}(\lambda)$ is the wavelength-dependent optical path difference (OPD) between two beams in the Michelson interferometer, which is given by

$$
\Delta_{M}(\lambda)=2 L+2 n(\lambda) t_{\text {eff }},
$$

where $2 L$ is the difference of path lengths between the interfering beams in the air whose dispersion is neglected and $n(\lambda)$ is the refractive index of the beam splitter material. The corresponding group OPD $\Delta_{M}^{g}(\lambda)$ is given by

$$
\Delta_{M}^{g}(\lambda)=2 L+2 N(\lambda) t_{e f f},
$$

where $N(\lambda)$ is the group refractive index.

\subsection{Michelson interferometer with a thin-film structure}

Next, let us consider that one of the mirrors of the interferometer is replaced by a thin-film structure on a substrate, which is characterized by a complex reflection coefficient

$$
r(\lambda)=\sqrt{R(\lambda)} \exp \left[i \delta_{r}(\lambda)\right]
$$

where $R(\lambda)$ is the reflectivity and $\delta_{r}(\lambda)$ is the phase change on reflection. The spectral signal $S(\lambda)$ corresponding to the spectra recorded at the output of the interferometer by the fibre-optic spectrometer can be expressed as

$$
\begin{aligned}
S(\lambda)= & V(\lambda) \exp \left\{-\left(\pi^{2} / 2\right)\left[\Delta^{g}(\lambda) \Delta \lambda_{R} / \lambda^{2}\right]^{2}\right\}, \\
& \times \cos [(2 \pi / \lambda) \Delta(\lambda)]
\end{aligned}
$$

where $V(\lambda)$ is a visibility term, which is given by

$$
V(\lambda)=V_{I} \frac{2 \sqrt{R(\lambda)}}{1+R(\lambda)}
$$

and $\Delta(\lambda)$ is the OPD given by

$$
\Delta(\lambda)=2 L+2 n(\lambda) t_{e f f}-\lambda \delta_{r}(\lambda) /(2 \pi)
$$

Moreover, we can construct, for the chosen mirror position $L=L_{0}$, the phase function $\delta(\lambda)$, which is referred to as the nonlinear-like phase [11] and which satisfies the relation

$$
\delta(\lambda)=(2 \pi / \lambda)\left\lfloor 2 L_{0}+2 n(\lambda) t_{\text {eff }}-\Delta(\lambda)\right\rfloor .
$$

\section{Experimental set-up}

The experimental set-up used in the application of whitelight spectral interferometry to measure the effective thickness of a beam splitter cube or the phase function of a thin-film structure is shown in Fig. 1. It consists of a white-light source, a halogen lamp HL-2000 (Ocean Optics, Inc.) with launching optics, an optical fibre and a collimating lens, a bulk-optic Michelson interferometer with a beam splitter cube made of BK7 optical glass (BS013, Thorlabs), metallic mirror 1 or a thin-film structure on a substrate, metallic mirror 2 connected to a micropositioner, a microscope objective, micropositioners, a read 
optical fibre, a miniature fibre-optic spectrometer S2000 (Ocean optics, Inc.), an A/D converter and a personal computer. The collimated beam of the white light has a diameter of approximately $3 \mathrm{~mm}$. The spectral interferograms are recorded at room temperature when the position of mirror 2 in the interferometer is adjusted with a precision of $1 \mu \mathrm{m}$.

The thin-film structure is represented by a uniform $\mathrm{SiO}_{2}$ thin film on a silicon wafer. $\mathrm{The}^{\mathrm{SiO}} 2$ thin film on the silicon wafer is prepared using a dry oxidation process described by the so-called Deal-Grove model [18]. Singlecrystal silicon wafer from ON Semiconductor, Czech Republic, is characterized by subsequent parameters, the diameter $(100 \pm 0.5) \mathrm{mm}$, orientation (111), B doped type $\mathrm{P}$, thickness $(381 \pm 25) \mu \mathrm{m}$ and resistivity $(0.008-0.009) \Omega \mathrm{cm}$. Before the oxidation, the wafer is cut into $40 \times 40 \mathrm{~mm}$ squares, cleaned by standard methods and then annealed in a furnace at $1200^{\circ} \mathrm{C}$. According to the model, the annealing time is selected in order to prepare $\mathrm{SiO}_{2}$ thin film with a thickness of approximately $340 \mathrm{~nm}$.

\section{Experimental results and discussion}

Prior to measurements, an efficient and accurate alignment of the beam splitter cube in the interferometer is achieved by observing the interference fringes when a laser diode instead of the white-light source is used. The effective thickness $t_{\text {eff }}$ of the beam splitter cube is measured by a technique based on the phase retrieval from the recorded spectral interferograms [13]. From the channelled spectrum $I_{M}(\lambda)$ and the reference spectrum $I^{(0)}{ }_{M}(\lambda)$, the spectral interference signal $S_{M}(\lambda)$ defined by Eq. (1) is determined. By processing the spectral signal using a WFT, the absolute OPD $\Delta_{M}(\lambda)$ between interfering beams is retrieved and then from its dependence on the refractive index $n(\lambda)$ of BK7 glass at room temperature the beam splitter effective thickness $t_{\text {eff }}$ is determined [13]. As an example, the beam splitter effective thickness $t_{\text {eff }}=-47.35 \mu \mathrm{m}$ with a standard deviation of $0.01 \mu \mathrm{m}$ is determined for the adjusted displacement $\Delta L=L-L^{\prime}=20 \mu \mathrm{m}$ of mirror 2 from the reference position $L^{\prime}$ corresponding to zero group OPD. In the thickness, the contribution of the UV adhesive layer between the two prisms that comprise the beam splitter is included. We assume that the refractive index of the layer is the same as that of the prisms. The negative effective thickness of the beam splitter cube means that the corresponding dispersive plate is in the other arm of the interferometer than is depicted in Fig. 1.

The nonlinear-like phase function $\delta(\lambda)$ of the thin-film structure is measured by a technique presented in a previous paper [11]. From the channelled and reference spectra, the spectral interference signal $S(\lambda)$ is determined. By processing the spectral signal using a WFT, the absolute OPD $\Delta(\lambda)$ between interfering beams is retrieved and the parameter $2 L_{0}$ is determined by a procedure based on the fact that the dependence of the OPD $\Delta(\lambda)$ on the refractive index $n(\lambda)$ of BK7 glass at room temperature deviates minimally

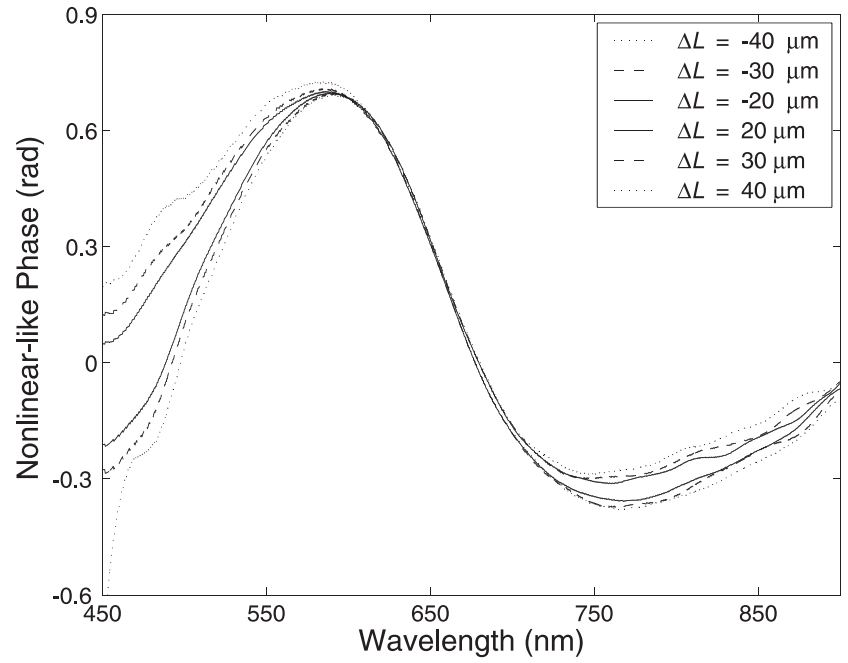

Fig. 2. Nonlinear-like phase as a function of wavelength for six different mirror displacements $\Delta L$ (the effective thickness $\left.t_{\text {eff }}=-47.35 \mu \mathrm{m}\right)$.

from linear dependence with the slope $2 t_{\text {eff }}=-94.70 \mu \mathrm{m}$. The nonlinear-like phase function $\delta(\lambda)$ is then simply determined by using Eq. (9). Figure 2 shows the nonlinear-like phase functions $\delta(\lambda)$ corresponding to six adjusted displacements $\Delta L=-40,-30,-20,20,30$, and $40 \mu \mathrm{m}$ of mirror 2 from the reference position $L$, which are the same as in the previous measurements and which are adjusted by another micropositioner connected to the thin-film structure. In other words, the mirrors of the interferometer have the same positions with respect to the beam splitter cube as in the previous measurements.

Figure 2 clearly demonstrates apparent discrimination between nonlinear-like phase functions with the upper ones for the positive displacements and shorter wavelengths whereas the lower functions at longer wavelengths are for the same displacements (right part of the figure is according to the legend). The discrimination can be attributed to the variable dispersion error as a result of the asymmetry in the interferometer or equivalently to the variable effective thickness of the beam splitter. To confirm this explanation, we measured the beam splitter effective thickness $t_{\text {eff }}$ for the all adjusted displacements of mirror 2. Figure 3 actually illustrates different thicknesses for different mirror positions that increase in the absolute value with increasing displacement. This effect is probably due to the alignment errors caused by relative rotation of the two prisms that comprise the beam splitter cube [16]. Finally, Fig. 4 shows the nonlinear-like phase functions measured for the all adjusted displacements of mirror 2 when the actual effective thickness of the beam splitter is taken into account. Figure 4 illustrates substantially better agreement between the nonlinear-like phase functions. However, it is clearly seen from Fig. 4 that the phase functions for the negative displacements (upper curves) have larger amplitude than those for the positive displacements. 


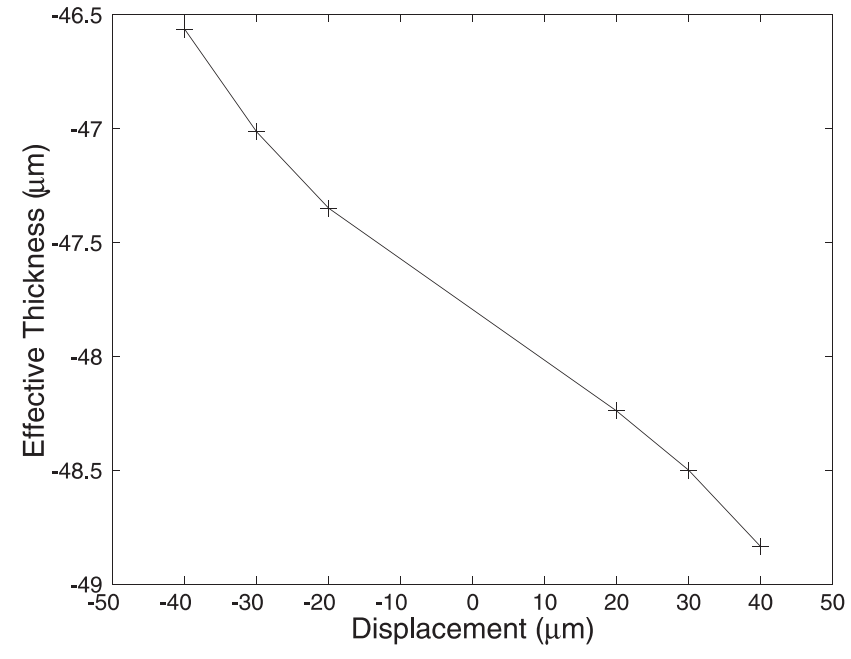

Fig. 3. Effective thickness of a beam splitter cube as a function of adjusted mirror displacement.

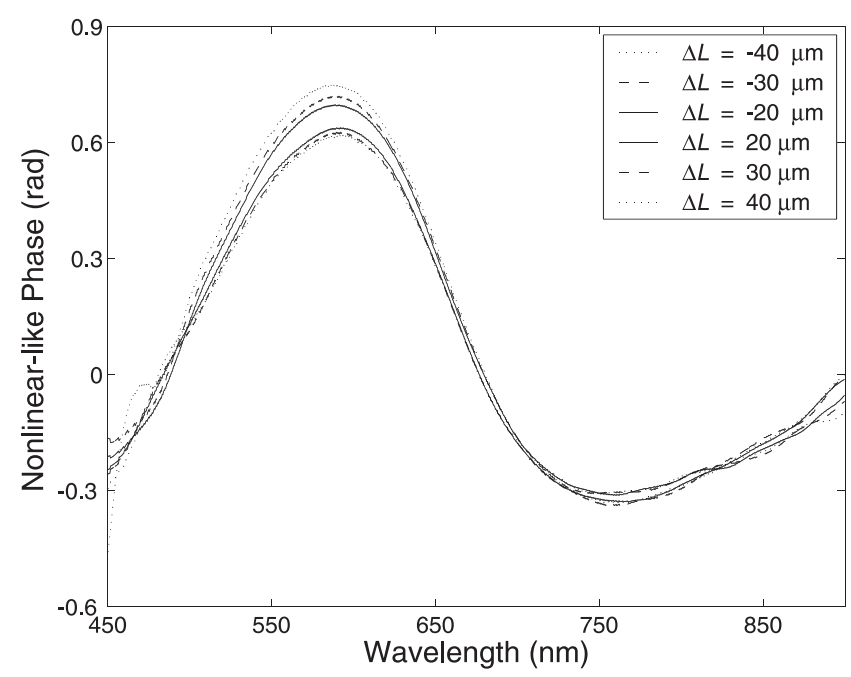

Fig. 4. Nonlinear-like phase as a function of wavelength for six different mirror displacements $\Delta L$ (the actual effective thicknesses are from Fig. 3).

\section{Conclusions}

We described a white-light spectral interferometric technique used to eliminate the dependence of the phase function of a thin-film structure on the path length difference adjusted in a Michelson interferometer. This phenomenon is due to a dispersion error of a commercially available beam splitter cube, the effective thickness of which varies with the adjusted path length difference (mirror position). The elimination of the dependence consisted of two steps. First, the Michelson interferometer with same metallic mirrors was used to measure the effective thickness of the beam splitter cube as a function of the path length difference. The effective thickness was obtained from the slope of the dependence of the phase on the refractive index of the beam splitter material (BK7 glass). Second, one of the mirrors of the Michelson interferometer was replaced by a thin-film structure and its nonlinear-like phase function was measured for the same path length differences as those adjusted in the first step.

We showed that the effective thickness of a beam splitter cube is an important parameter for an accurate interferometric measurement of the nonlinear-like phase function of a thin-film structure. The measurement, which can be extended to smaller diameters of convergent light beams and which has the primary advantage over a technique such as ellipsometry in its normal incidence configuration, is possible without the need of the microscope interferometer.

\section{Acknowledgements}

The research was partially supported by the Grant Agency of the Czech Republic (project no 202/06/0531), by grant MSM6198910016 and by internal grant of TU Ostrava (IGS HGF VŠB-TUO).

\section{References}

1. L.M. Smith and C.C. Dobson, "Absolute displacement measurement using modulation of the spectrum of white light in a Michelson interferometer", Appl. Optics 28, 3339-3342 (1989).

2. U. Schnell, E. Zimmermann, and R. Dändliker, "Absolute distance measurement with synchronously sampled whitelight channelled spectrum interferometry", J. Opt. A-Pure Appl. Op. 4, 643-651 (1995).

3. P. Hlubina, "Dispersive spectral-domain two-beam interference analysed by a fibre-optic spectrometer", J. Mod. Opt. 51, 537-547 (2004).

4. J. Schwider and L. Zhou, "Dispersive interferometric profilometer", Opt. Lett. 19, 995-997 (1994).

5. P. Sandoz, G. Tribillon, and H. Perrin, "High-resolution profilometry by using phase calculation algorithms for spectroscopic analysis of white-light interferograms", J. Mod. Opt. 43, 701-708 (1996).

6. A.F. Zuluaga and R. Richards-Kortum, "Spatially resolved spectral interferometry for determination of subsurface structure", Opt. Lett. 24, 519-521 (1999).

7. D. Reolon, M. Jacquot, I. Verrier, G. Brun, and C. Veillas, "Broadband supercontinuum interferometer for high-resolution profilometry", Opt. Express 14, 128-137 (2006).

8. U. Schnell, R. Dändliker, and S. Gray, "Dispersive whitelight interferometry for absolute distance measurement with dielectric multilayer systems on the target", Opt. Lett. 21, 528-530 (1996).

9. Y. Liang and C.H. Grover, "Modified white-light MachZehnder interferometer for direct group-delay measurements", Appl. Optics 37, 4105-4111 (1998).

10. I. Gurov, P. Hlubina, and V. Chugunov, "Evaluation of spectral modulated interferograms using a Fourier transform and the iterative phase-locked loop method", Meas. Sci. Technol. 14, 122-130 (2003).

11. P. Hlubina, D. Ciprian, J. Luňáček, and M. Lesňák, "Dispersive white-light spectral interferometry with absolute phase retrieval to measure thin film", Opt. Express 14, 7678-7685 (2006). 
12. D. Reolon, M. Jacquot, I. Verrier, G. Brun, and C. Veillas, "High resolution group refractive index measurement by broadband supercontinuum interferometry and wavelet-transform analysis", Opt. Express 14, 12744-12750 (2006).

13. P. Hlubina, J. Luňáček, D. Ciprian, and R. Chlebus, "Windowed Fourier transform applied in the wavelength domain to process the spectral interference signals", Opt. Commun. 281, 2349-2354 (2008).

14. K.B. Farr and N. George, "Beamsplitter cube for white light interferometry", Opt. Eng. 31, 2191-2196 (1992).

15. A. Pfärtner and J. Schwider, "Dispersion error in white-light Linnik interferometers and its applications for evaluation procedures", Appl. Opt. 34, 6223-6228 (2001).
16. P.D. Koudelka and J.H. Burge, "Fabrication of cube beamsplitter for white light interferometry", Proc SPIE 5252, 17-25 (2004)

17. P. Hlubina, "White-light spectral interferometry to measure the effective thickness of optical elements of known dispersion", Acta Phys. Slovaca 55, 387-393 (2005).

18. J.D. Plummer, M.D. Deal, and P.B. Griffin, Silicon VLSI Technology - Fundamentals, Practice and Modelling, Prentice Hall, Upper Saddle River, 2000.

19. Schott Computer Glass Catalogue 1.0, Schott Glasswerke, Mainz, 1992. 